\title{
"smarter medicine»: la liste «Top-5» de la SSC
}

\section{Organisation responsable "smarter medicine»}

Depuis quelques années, la Société suisse de chirurgie (SSC) s'engage pour que la formation postgraduée et continue des chirurgiens permette, grâce à des travaux et des mesures scientifiques de la qualité des traitements, une prise en charge de la population avec les standards de qualité les plus élevés. A notre époque, des solutions doivent être trouvées pour augmenter la productivité sans altérer la qualité des traitements. A cet égard, la SSC considère l'initiative "Choosing Wisely" et les recommandations de "smarter medicine» comme une possibilité adéquate de se rapprocher de cet objectif.

Pour le domaine de la chirurgie, cette liste Top-5 a été élaborée par l'American College of Surgeons en collaboration avec différents partenaires. La SSC s'est appuyée sur la liste américaine et l'a jugée intégralement applicable et pertinente pour la Suisse.

La Société Suisse de Chirurgie recommande de ne pas pratiquer les interventions suivantes en chirurgie:

1. Ne pas effectuer de curage axillaire aux stades cliniques I et II du cancer du sein si les ganglions lymphatiques sont cliniquement normaux, sans avoir procédé auparavant à une biopsie du ganglion sentinelle.

La biopsie de ganglion sentinelle a prouvé son efficacité pour explorer l'aisselle à la recherche de ganglions lymphatiques anormaux, et il est également prouvé qu'elle a moins d'effets secondaires à court et à long terme; elle est notamment associée à un risque notablement plus faible de lymphœdème (gonflement permanent du bras).

Si le(s) ganglion(s) lymphatique(s) ne se révèle(nt) pas cancéreux, aucun curage axillaire ne devrait être effectué.

Si un ou deux ganglions sentinelles sont envahis par un cancer non invasif, que la patiente a eu une chirurgie mammaire conservatrice et qu'une radiothérapie complète du sein est prévue avec programmation d'un traitement systémique approprié, aucun curage axillaire ne devrait être effectué.

\section{La campagne "smarter medicine»}

Une nouvelle association de soutien dynamise en Suisse l'initiative "smarter medicine» lancée il y atrois ans. Les orientations de la campagne sont soutenues par des organisations médicales spécialisées et professionnelles, mais aussi par des associations qui défendent les intérêts des patients et des consommateurs. Ensemble, elles souhaitent sensibiliser le public au fait qu'en médecine et pour certains traitements, moins peut parfois signifier plus de qualité de vie pour les personnes concernées. «Smarter medicine» s'est adaptée à l'initiative américaine "Choosing Wisely" qui remporte un grand succès et qui a pour but non seulement de déboucher sur des "décisions judicieuses", mais aussi d'encourager une franche discussion entre le corps médical, les patients et le public.

Dans les prochains mois, d'autres sociétés de disciplines publieront leurs listes Top- 5 avec des recommandations pour leurs domaines d'activité.

Vous trouverez plus d'informations concernant l'asscociation de soutien et les listes Top-5 publiées sous www.smartermedicine.ch.

2. Eviter la tomodensitométrie «corps entier» systématique chez les patients présentant un traumatisme mineur ou localisé.

Un usage agressif de la tomodensitométrie «corps entier» améliore le diagnostic précoce de lésion et peut même avoir un impact positif sur la survie de patients polytraumatisés. Néanmoins, il convient de prendre en compte l'exposition significative aux radiations ainsi que les coûts qu'impliquent ces examens, notamment chez les patients dont la lésion est secondaire à un traumatisme à faible énergie en l'absence de données cliniques en faveur d'un traumatisme majeur.

3. Eviter les tests de dépistage du cancer colorectal chez les patients asymptomatiques ayant une espérance de vie inférieure à dix ans sans antécédents familiaux ou personnels de néoplasie colorectale. Il a été démontré que le dépistage du cancer colorectal réduisait la mortalité associée à cette maladie répandue; la coloscopie permet de détecter et de retirer les polypes adénomateux, la lésion précancéreuse de nombreux cancers, réduisant ainsi l'incidence de la maladie plus tard au cours de la vie. 


\section{Elaboration de cette liste}

L'American College of Surgeons (ACS) a prié la Commission on Cancer, le Committee on Trauma et les Advisory Councils for Colon and Rectal Surgery, General Surgery and Pediatric Surgery d'établir des recommandations en lien avec la campagne Choosing Wisely de I'American Board of Internal Medicine (ABIM). Une description des objectifs de la campagne et un lien vers le site Choosing Wisely ont été fournis aux comités; les recommandations publiées par des organisations participant déjà à la campagne ont été référencées et révisées pendant les débats. Toutes les recommandations rassemblées par les comités de l'ACS ont été révisées, et cinq éléments ont été identifiés. La Société suisse de Chirurgie s'est appuyé sur la liste "Choosing Wisley" publiée par l'American College of Surgeons et I'a jugée intégralement applicable et pertinente pour la Suisse.

Une liste détaillée des références et des recommandations d'autres sociétés médicales sont disponibles en ligne sous www. smartermedicine.ch

Néanmoins, les modalités de dépistage et de surveillance sont inappropriées si les risques l'emportent sur les avantages.

Le risque lié à la coloscopie augmente avec l'âge et les comorbidités.

Le ratio risques/avantages du dépistage et de la surveillance du cancer colorectal relatif à chaque patient devrait être défini de manière personnalisée sur la base des données cliniques antérieures, des antécédents familiaux, du risque estimé de l'intervention, de l'espérance de vie et des préférences du patient.

4. Eviter la radiographie thoracique à l'admission ou en préopératoire chez les patients en ambulatoire sans antécédents particuliers et dont l'examen clinique est normal.

Il n'est pas recommandé pour les patients en ambulatoire de procéder systématiquement à une radiographie thoracique à l'admission ou en préopératoire sans indication spécifique en relation avec les antécédents et/ou les résultats de l'examen clinique. Seules $2 \%$ des radiographies effectuées entrainent une modification de la prise en charge du patient. Une radiographie thoracique peut être justifiée en cas de suspicion de pathologie cardio-pulmonaire ou s'il y a des antécédents de pathologies cardio-pulmonaires stables chroniques chez des patients de plus de 70 ans n'ayant pas eu de radiographie thoracique au cours des six derniers mois.

5. Ne pas effectuer de tomodensitométrie pour examiner des enfants qui présentent une suspicion d'appendicite tant que l'échographie n'a pas été réalisée.

Même si la tomodensitométrie est efficace pour détecter une appendicite suspectée chez les enfants, l'échographie est l'examen d'imagerie de choix chez les enfants. Si les résultats de l'examen échographique sont équivoques, celui-ci peut être suivi d'une tomodensitométrie. Cette approche est efficace en termes de coûts, réduit les risques de radiations potentielles et elle est extrêmement précise, avec une sensibilité et une spécificité s'élevant actuellement à $94 \%$ entre des mains expérimentées. Pour améliorer le diagnostic sans user de la tomodensitométrie - sachant que l'expérience individuelle des opérateurs est variable -, on peut envisager raisonnablement des formations visant à améliorer l'expérience des opérateurs en matière d'échographie adaptée aux différentes communautés et établir des règles de décision clinique basées sur des preuves. 